\title{
Supporting Dynamic Spectrum Access in Heterogeneous LTE+ Networks
}

\section{Special Interest Group on Data Communication}

The INL is a

U.S. Department of Energy

National Laboratory

operated by

Battelle Energy Alliance

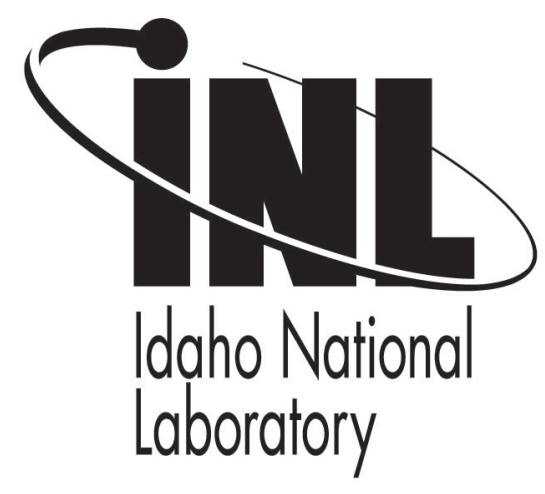

\author{
Juan D. Deaton \\ Mike Benonis \\ Luiz DaSilva \\ Ryan E. Irwin
}

\section{August 2012}

This is a preprint of a paper intended for publication in a journal or proceedings. Since changes may be made before publication, this preprint should not be cited or reproduced without permission of the author. This document was prepared as an account of work sponsored by an agency of the United States Government. Neither the United States Government nor any agency thereof, or any of their employees, makes any warranty, expressed or implied, or assumes any legal liability or responsibility for any third party's use, or the results of such use, of any information, apparatus, product or process disclosed in this report, or represents that its use by such third party would not infringe privately owned rights. The views expressed in this paper are not necessarily those of the United States Government or the sponsoring agency. 


\section{Supporting Dynamic Spectrum Access in Heterogeneous LTE+ Networks}

\author{
Juan D. Deaton*† \\ *N\&HS Directorate \\ Idaho National Lab \\ Idaho Falls, Idaho USA
}

\author{
Mike Benonis ${ }^{\dagger}$ \\ $\dagger$ Wireless@VT \\ Bradley Dept. of ECE \\ Virginia Tech \\ Blacksburg,Virginia USA
}

\author{
Luiz DaSilva \\ $\ddagger$ CTVR \\ Trinity College \\ Dublin, Ireland
}

\author{
Ryan E. Irwin ${ }^{\dagger \S}$ \\ $\S$ Raytheon BBN Technologies \\ Advanced Network Systems \\ Cambridge, MA USA
}

\begin{abstract}
With Long Term Evolution Advanced (LTE+) networks likely as the future one world 4G standard, network operators may need to deploy a Dynamic Spectrum Access (DSA) overlay in Heterogeneous Networks (HetNets) to extend coverage, increase spectrum efficiency, and increase the capacity of these networks. In this paper, we propose three new management frameworks for DSA in an LTE+ HetNet: Spectrum Accountability Client, Cell Spectrum Management, and Domain Spectrum Management. For these spectrum management frameworks, we define protocol interfaces and operational signaling scenarios to support cooperative sensing, spectrum lease management, and alarm scenarios for rule adjustment. We also quantify, through integer programs, the benefits of using DSA in an LTE+ HetNet that can opportunistically reuse vacant TV and GSM spectrum. Using integer programs, we consider a topology using Geographic Information System data from the Blacksburg, VA metro area to assess the realistic benefits of DSA in an LTE+ HetNet.
\end{abstract}

\section{INTRODUCTION}

With at least 20 commercial deployments since 2009, Long Term Evolution (LTE) has the fastest adoption rate of any mobile network technology to date. More than 200 operators in 80 countries are investing in LTE, positioning LTE as the future global standard for mobile wireless technologies [1]. Despite the rapid deployment of LTE networks, the Federal Communications Commission (FCC) predicts that the rapid user adoption of smart phone devices and applications will create a $300 \mathrm{MHz}$ spectrum deficit for mobile wireless broadband by 2014 [2]. As a result of this spectrum deficit, network operators must find new ways to increase the capacity of their LTE networks.

Supporting the network operators, regulatory agencies and standards bodies are also seeking solutions to the spectrum crisis. In a separate report, the FCC found that spectrum

This work supported by the Idaho National Laboratory (INL) Ph.D. Candidate Program and Virginia Tech Bradley Fellowship. Work supported through the INL is supported through the Laboratory Directed Research \& Development (LDRD) Program under DOE Idaho Operations Office Contract DE-AC07-05ID14517. The United States Government retains and the publisher, by accepting the article for publication, acknowledges that the United States Government retains a nonexclusive, paid-up, irrevocable, worldwide license to publish or reproduce the published form of this manuscript, or allow others to do so, for United States Government purposes. The views and conclusions contained in this document are those of the authors and should not be interpreted as representing the official policies, either expressed or implied, of the Department of Energy or the U.S. Government. is mostly underutilized throughout time and space [3]. Accordingly, rigid regulatory paradigms for spectrum management are shifting toward more flexible Dynamic Spectrum Access (DSA) models [4, 5], where primary spectrum can be accessed dynamically by secondary users when not in use. Along this same line, Long Term Evolution Advanced (LTE+) standards now support spectrum aggregation to increase transmission bandwidths (i.e., carriers of up to $100 \mathrm{MHz}$ through the aggregation of discontiguous spectrum channels) [6]. Using spectrum aggregation, a combination of licensed and DSA channels could be deployed on cell carriers, i.e., a hybrid carrier [7], to expand capacity. To extend coverage, increase spectrum efficiency, and augment capacity, LTE+ standards also support Relay Nodes (RNs) and femto-cell sized Evolved Node Bs (eNBs), called Home Evolved Node Bs (HeNBs). Operators hope to increase their capacity by building LTE+ Heterogeneous Networks (HetNets), including $\mathrm{RNs}$ and HeNBs, which can be deployed in institutional settings (e.g., stadiums, campuses, shopping centers, etc.), improving customer service and generating additional revenue. Recognizing these trends, our work examines the architectural and operational aspects of deploying a DSA overlay in LTE HetNets.

Additionally, with the advent of LTE+, network operators may wish to opportunistically reuse their own spectrum from Global System for Mobile Communications (GSM) networks to augment LTE+ spectrum. According to 4G Americas, GSM still has $77 \%$ worldwide penetration and its use will decrease as LTE+ networks increase [8]. Therefore, it may be cogent to opportunistically use GSM frequencies for LTE+ carriers before GSM spectrum is refarmed (i.e., while operators are still supporting legacy GSM devices). Techniques for the opportunistic usage of GSM frequencies by Worldwide Interoperability for Microwave Access (WiMax) networks are proposed in [9]. Through predicting demand and spectrum vacancies, future LTE+ networks could dynamically self-configure, request, and assign spectrum resources through hybrid carriers. In our work, we simulate vacant GSM channels, as well as TV white spaces, being opportunistically used by an LTE+ network.

In our previous work, we analyzed the effects of DSA in a single-tiered LTE+ network (MacroNet) and proposed the Spectrum Accountability (SA) architectural framework to 
support registration, neighbor discovery, cooperative sensing, and detection and enforcement of spectrum access rules [17]. Our work in [17] extended many concepts from IEEE 1900.4a and 1900.5 through providing methods for monitoring use of spectrum leases, detecting violations, resolving conflicts, and modifying spectrum usage policies within the scope of an LTE+ network. In summary, SA is a framework by which regulators can define, enforce, and manage spectrum access rules for competitive secondary operators and primary operators. In this paper, we examine the architectural and operational impacts of SA framework when applied to an LTE+ HetNet.

Most HetNet research focuses on cross-tier interference (i.e., interference between femto-cell and macro cell layers) [10-14]. Many works proposing these algorithms assume an architectural and regulatory framework to support DSA for cognitive femto-cells, to support opportunistic spectrum use. Work in $[15,16]$ both propose spectrum management architectures and discuss fundamental research issues related to femto-cells. However, these works did not explore how the network would manage the combined primary and secondary usage of spectrum, nor the architecture and signaling that would be required for the network to support this kind of operation. Additionally, while it is generally understood femtocells can improve capacity, quantifying and comparing the benefits between macro-cell and femto-cell networks using opportunistic spectrum has not been performed.

This paper makes the following contributions. We identify and propose three DSA management frameworks for LTE+ HetNets: Spectrum Accountability Client, Cell Spectrum Management, and Domain Spectrum Management. Using our management frameworks, we define protocol interfaces and operational signaling scenarios to support cooperative sensing, spectrum lease management, and alarm scenarios for rule adjustment. We believe these spectrum management frameworks could serve as a guide for future LTE+ HetNet standards using DSA. While we focus on the LTE+ HetNet in this paper, we argue that any HetNet that uses DSA can also adopt the management frameworks that we propose. In our second contribution, we formulate integer programs to quantify benefits of deploying DSA spectrum in an LTE+ HetNet. Using these programs, we can compare optimal channel assignment for an LTE+ HetNet that uses only licensed spectrum against another that takes advantage of hybrid carriers using opportunistic GSM and TV white space spectrum. In our final contribution, we model a HetNet topology using population data, building footprints, existing transmitter locations, and FCC allocated spectrum bandwidths to assess the realistic benefits of using DSA in an LTE+ HetNet. We believe that using this Geographic Information System (GIS) data in our final contribution produces more detailed, richer, and more realistic inputs for our integer programs.

Our paper is organized as follows. Section II introduces the LTE+ HetNet and illustrates the architectural effects of introducing a DSA overlay. The section also introduces our three proposed architectural frameworks for managing DSA spectrum in an LTE+ HetNet architecture. We name these frame-

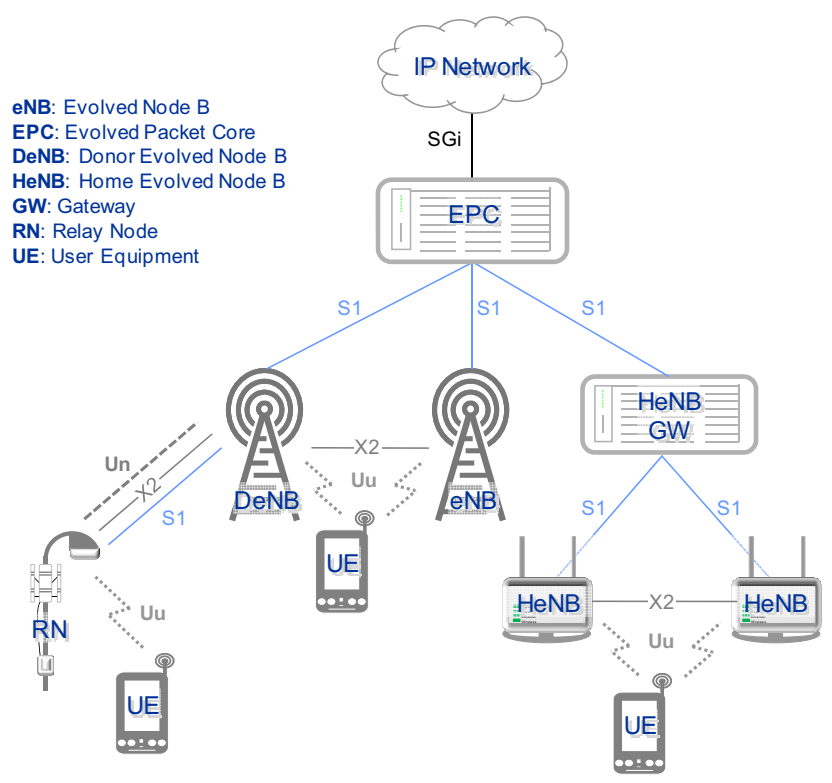

Fig. 1. The addition of the Relay Node (RN) and Home Evolved Node B (HeNB) to the standard LTE architecture creates the LTE+ HetNet architecture.

works Spectrum Accountability Client (SAC), Cell Spectrum Management (CSM), and Domain Spectrum Management (DSM). In Section III, we show the operational effects of each configuration through selected signaling diagrams. In Section IV, we model an LTE+ HetNet through integer programs and use these models, in conjunction with GIS data, to create an input topology for assessing the quantitative benefits of using DSA. We then conclude the paper in Section V, where we propose new research questions and summarize our results.

\section{Effects of DSA in the LTE+ HetNet Architecture}

In this section, we provide an architectural overview of the effects of a DSA overlay in the LTE+ HetNet architecture. This section first presents a brief overview of the LTE+ HetNet architecture. Next, using the SA framework from [17], we propose new network elements, interfaces, and functionality in the LTE+ HetNet to support DSA. At the end of this section, we introduce our three proposed DSA management frameworks for LTE+ HetNets: SAC, CSM, and DSM.

\section{A. The LTE+ HetNet}

Figure 1 illustrates the LTE+ HetNet architecture (it also includes an acronym list for convenience). In LTE + , the User Equipment (UE) is the end user, which only has access to packet-switched services (i.e., no circuit switched voice). Through the LTE+ air interface (LTE-Uu), the UE connects to the LTE+ network using the eNB, where the eNB has the important function of Radio Resource Control (RRC). RRC is responsible for the establishment, configuration, maintenance, and release of radio bearers. The $\mathrm{X} 2$ link is used for communication between eNBs to assist in handoffs between neighboring eNBs and in the exchange of signaling information. The Evolved Packet Core (EPC) is a combination of many 


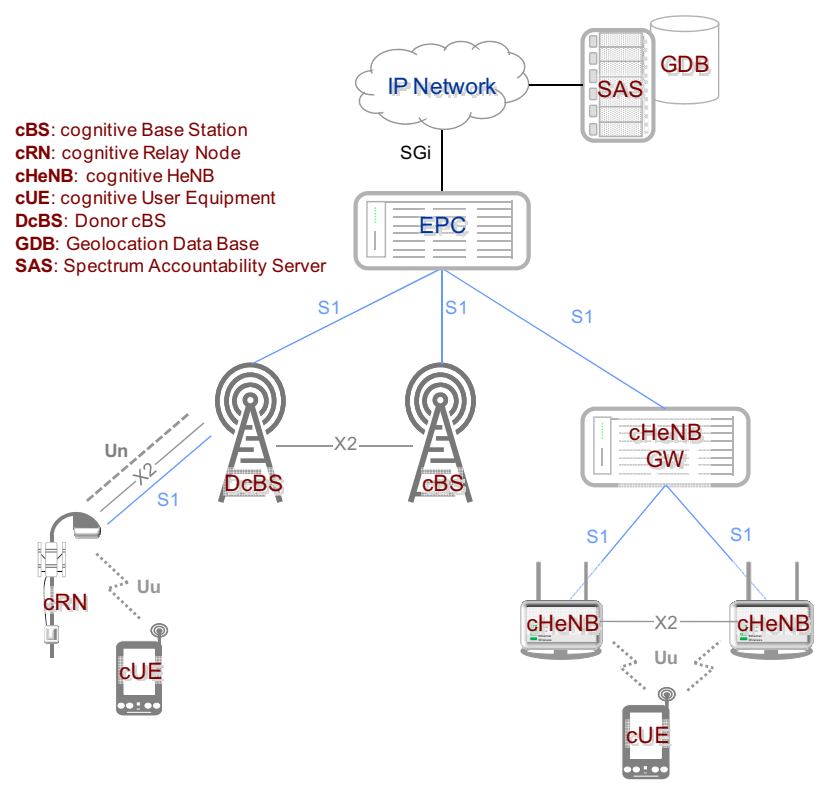

Fig. 2. The introduction of DSA within the LTE+ HetNet introduces cognitive network elements and the Spectrum Accountability Server (SAS).

network elements that authorizes network access, coordinates data bearers, manages mobile addressing, and provides an IP anchor for UEs to communicate with external network hosts. The $\mathrm{S} 1$ is used to carry control information as well as user traffic from the eNB to the EPC. S1 is also capable of carrying signaling information among the eNBs.

In LTE, a HetNet is formed through the introduction of either of two new network elements: the Relay Node (RN) and the Home Evolved Node B (HeNB). LTE+ supports relaying through RNs, which extend coverage and use an eNB, known as the Donor Evolved Node B (DeNB), for backhaul. RNs appear to UEs as an eNB, while supporting a unique interface to the DeNB called the Un. The DeNB serves as an EPC proxy to the RN by embedding S1 and X2 over the Un to the RN (See Figure 1). Femto-cells are supported through network elements called the HeNBs and HeNB Gateways. The HeNB supports eNB functionality with a femto-sized cell coverage and can use the premises infrastructure for backhaul. HeNBs may use an optional HeNB Gateway to serve as an S1 concentrator and relay between many HeNBs and the EPC. Although the HeNB is connected to the premises IP network, all user traffic from the UEs uses an IP anchor at the EPC (i.e., all user traffic flows through the core network). HeNBs also support X2 links to other HeNBs for handoffs. While this brief introduction to LTE+ is sufficient to support further discussion in this paper, the reader is encouraged to consult $[18,19]$ for more detailed information.

\section{B. DSA in the LTE+ Heterogeneous Network}

We propose to support the DSA overlay through the introduction of the following network elements: the Spectrum Accountability Server (SAS), cognitive Base Station (cBS), cognitive User Equipment (cUE), cognitive Relay Node (cRN),

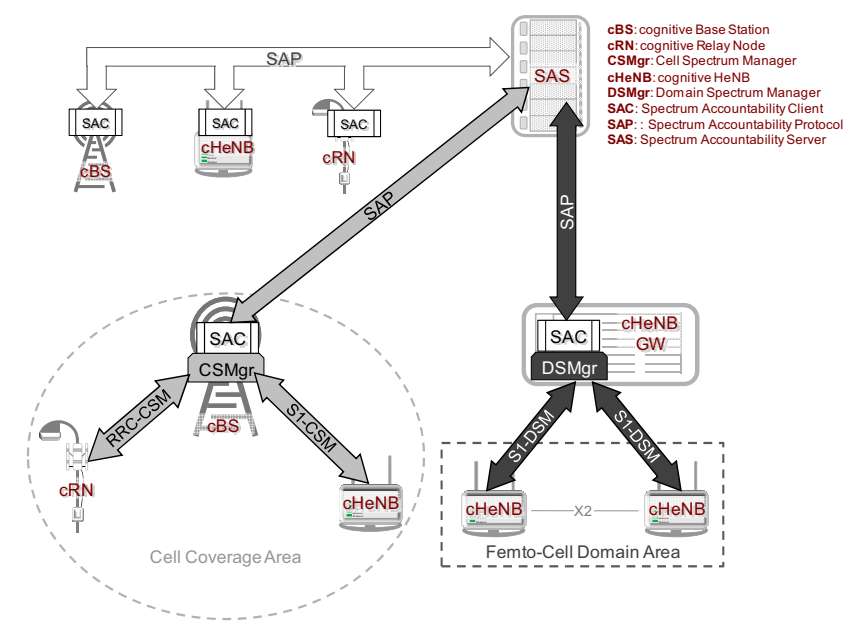

Fig. 3. The use of DSA within the LTE+ HetNet introduces three options for spectrum resource management: Spectrum Accountability Client (SAC) only (top left), Cell Spectrum Management (CSM) (bottom left), and Domain Spectrum Management (DSM) (bottom right).

and the cognitive Home evolved Node B (cHeNB). These network elements are shown in Figure 2. The SAS manages spectrum access policies and monitors spectrum leases through Key Performance Indicators (KPI) reported through the leasing entities (e.g., cBS, cHeNB, and cRN). Through an external IP address, the leasing entities register with the SAS, discover neighbors, exchange sensing information, request leases, and report KPI to the SAS. The cUE carries all the same functionality as the UE. However, the cUE also has a spectrum agile radio, capable of operating on and sensing multiple bands as directed by the network. The cRN and cHeNB both have similar functionality, albeit more limited, as a cBS (e.g., spectrum sensing, SAS client, RRC, etc.); however, functionality can vary depending on the management framework for the HetNet.

\section{DSA Management Frameworks for the LTE+ Heteroge- neous Network}

Extending our work from [17], we propose three different spectrum management frameworks for the LTE+ HetNet: SAC only, CSM, and DSM. Network elements impacted by these management frameworks are indicated in Figure 3. In the SAC only framework, shown in Figure 3 top left, both the cRN and the cHeNB behave like a cBS in the SA framework and communicate directly to the SAS. Spectrum leases are managed directly through the SAS and deployed as carrier resources for $\mathrm{cRNs}$ and $\mathrm{cHeNBs}$ without any interaction from other network elements. In contrast to SAC, in CSM, shown in Figure 3 bottom left, the cRNs and $\mathrm{cHeNBs}$ request spectrum resources from the Cell Spectrum Manager (CSMgr) instead of from the SAS. In CSM, the CSMgr requests spectrum leases from the SAS and manages a local spectrum pool for the cRNs and $\mathrm{cHeNBs}$ within the cell coverage area. DSM is similar to CSM; however, in DSM the cHeNB Gateway serves as the local spectrum manager for the domain where the cHeNBs reside. In DSM, Figure 3 bottom right, only cHeNBs are 

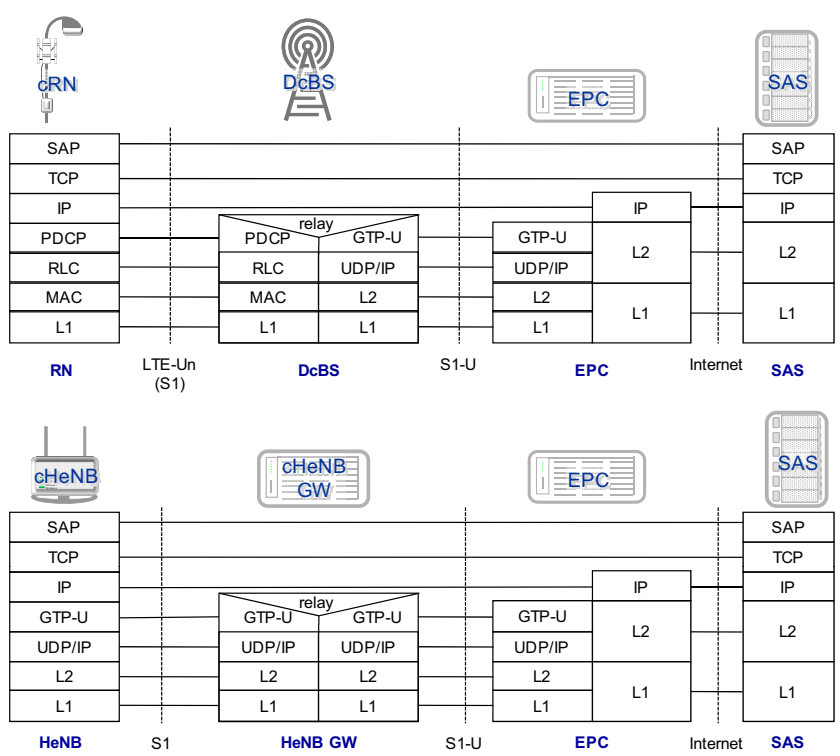

Fig. 4. When using a SAC configuration to support DSA within the LTE+ HetNet, the DcBS and the cHeNB Gateway serve as proxies for the $\mathrm{cRN}$ and cHeNB, respectively, to communicate with the SAS.

assigned spectrum resources through the Domain Spectrum Manager (DSMgr). In the sequel, we present each of these spectrum management options individually and discuss their operational and interface effects on the cRN and cHeNB.

\section{DSA Spectrum Frameworks}

\section{A. Spectrum Accountability Client Only}

When the SAC framework only is used, cRNs and cHeNBs communicate directly with the SAS for spectrum leases. In these cases, the DcBS and the cHeNB Gateway serve as proxies for the cRNs and cHeNBs, respectively. As with the cBS, both the $\mathrm{cRN}$ and $\mathrm{cHeNB}$ have IP anchors supported by the EPC to enable communication with external networks. This functionally serves as the basis for the Spectrum Accounting Protocol (SAP), which supports the DSA service request and spectrum management procedures [17]. The protocol stacks and interfaces for each network element to support SAP for the SAC configuration are shown in Figure 4. For the SAC configuration, DSA supporting procedures from [17] remain unchanged, because the cRN and cHeNB are seen exactly as cBSs.

\section{B. Cell Spectrum Management}

In contrast to SAC, CSM provides local spectrum management of the coverage area of the cBS, through a logical entity known as the CSMgr. All cRNs and cHeNBs that reside in the geographic coverage area of the cBS are assigned spectral resources by the CSMgr. Spectrum resources are managed through a local spectrum pool, which the CSMgr assigns to the cRNs and cHeNBs within the cell coverage area. When the demand within the cell exceeds the available spectral capacity, the cBS, through its own Spectrum Accountability Client (SAC) and the CSMgr, will generate a lease request or obtain more spectrum for the spectrum pool.

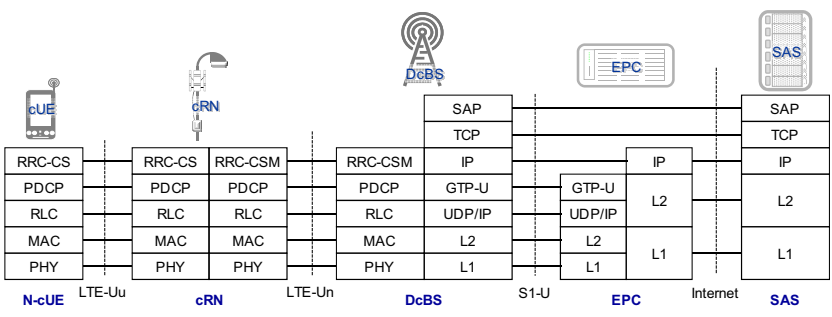

Fig. 5. Radio Resource Control-Cell Spectrum Management (RRC-CSM) signaling is used to locally manage the cRN in CSM, where the Cell Spectrum Manager (CSMgr), on the DcBS, translates orders and information to and from other $\mathrm{cBS}$ and the SAS. The Radio Resource Control-Cooperative Sensing (RRC-CS) coordinates spectrum sensing signaling between the UE and the $\mathrm{RN}$.

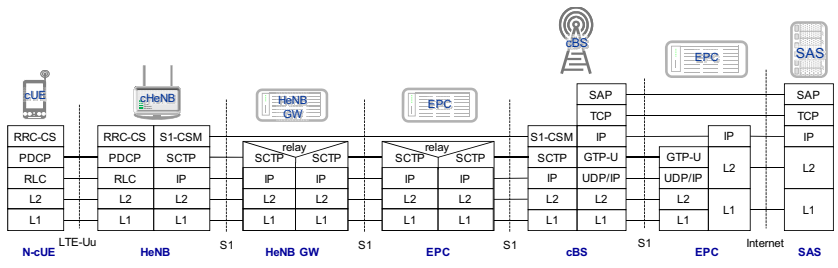

Fig. 6. S1-CSM signaling is used to locally manage the cHeNB in CSM, where the Cell Spectrum Manager (CSMgr), on the cBS, translates orders and information to and from other $\mathrm{CBS}$ and the SAS. The RRC-CS coordinates spectrum sensing signaling between the UE and the cHeNB.

1) CSM Interfaces: The CSMgr communicates directly with the cRN using the RRC-CSM protocol, over the Un interface, but uses the cHeNB Gateway and EPC as relays for communication between the cHeNB and the cBS. The protocol stack showing the DSA interfaces for the cRN and the other network elements is shown in Figure 5. When the CSM configuration is used with the cRN, the cRN receives orders from the DcBS for spectrum sensing and then translates those sensing orders to the cUEs for which it provides service. Spectrum resource requests are performed using the RRCCSM protocol over the Un interface. The resource requests are evaluated by the spectrum manager and then translated into spectrum lease requests to the SAS to increase the cell capacity when needed. Similarly, the cHeNB also receives orders for sensing and sends requests for resources to the CSMgr. The protocol stack showing the DSA interfaces for the cHeNB and the other network elements is shown in Figure 6. However, in the case of the $\mathrm{cHeNB}$, this signaling is performed over the S1-CSM interface, which uses relay functions on the cHeNB Gateway and the EPC. We also note that the RRCCSM protocol is a possible option for cHeNBs as well.

2) CSM Procedures: In the CSM configuration, the CSMgr translates orders and information between the $\mathrm{cRN}$ and $\mathrm{cHeNB}$ within the local cell and the SAS and other cBSs. We illustrate how this translation occurs through modifying the following procedures from [17]: cooperative sensing, spectrum lease request, and new primary operator alerting procedure. Operationally, these procedures are identical for the cRN and the cHeNB. The primary difference is the interface between the $\mathrm{cRN} / \mathrm{cHeNB}$ and the cBS. In the interfaces that we have 


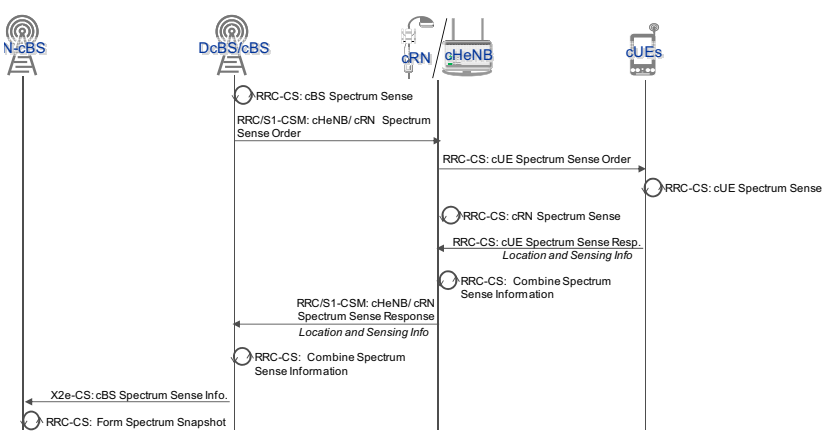

Fig. 7. Cooperative sensing procedure for both the $\mathrm{cRN}$ and the $\mathrm{cHeNB}$ in the Cell Spectrum Management (CSM) configuration.

described, we assume the RRC-CSM for the cRN and the S1CSM for the cHeNB in the subsequent procedures.

a) CSM Cooperative Sensing Procedure: When more thorough knowledge of spectral conditions is required, cooperative sensing can be used. The cooperative sensing procedure is illustrated in Figure 7. In the cooperative sensing procedure, the CSMgr on the DcBS/cBS sends spectrum sensing orders to $\mathrm{cRNs} / \mathrm{cHeNBs}$ for the collection of spectrum sensing information. The cRNs/cHeNBs translate the spectrum sensing orders to the cUEs that they serve, and the cUEs perform local sensing. After all cUEs have returned their sensing response, the cRNs/cHeNBs combine this sensing information and send it to the $\mathrm{DcBS} / \mathrm{cBS}$, in the spectrum sensing responses. After receiving this response, the $\mathrm{DcBS} / \mathrm{cBS}$ combines this information and shares it with the Neighboring-cognitive Base Station $(\mathrm{N}-\mathrm{cBS})$ or the SAS.

b) CSM Spectrum Lease Request Procedure: When the cRNs/ cHeNBs encounter the need for more spectrum (i.e., a trigger event) they send spectrum requests to the DcBS/cBS. This signaling procedure is illustrated in Figure 8. Trigger events are the result of traffic trending/prediction algorithms, which could detect and predict sharp rises in traffic from an unexpected event or a more gradual increase in load. In our example, the $\mathrm{cRN} / \mathrm{cHeNB}$ does not have sufficient spectral resources to serve the spectral needs of its cell and sends resource requests to the DcBS/cBS, using the RRC/S1CSM. Based on the local needs of the cell, the DcBS/cBS calculates the resources needed and then forms a spectrum lease request and sends that lease request to the SAS. The SAS evaluates the spectrum availability and then issues the lease to the DcBS/cBS. After receiving the spectrum lease, the DcBS/cBS calculates the resource assignment for the local cell and assigns the specific spectral resources to each of the $\mathrm{cRNs} / \mathrm{cHeNBs}$ requesting resources. Using these spectral resources, the cRNs/cHeNBs service the requests of the associated cUEs. Once the lease has expired, a notification is sent from the SAS to the DcBS/cBS to release the spectral resources. The $\mathrm{cRN} / \mathrm{cHeNB}$ then translates this information into spectral resource orders for the local cell. An Acknowledgment $(\mathrm{ACK})$ is returned from each of the $\mathrm{cRNs} / \mathrm{cHeNBs}$ to the $\mathrm{DcBS} / \mathrm{cBS}$ indicating a release of the spectrum as well as the KPI of the spectrum use. After all the spectrum release ACKs

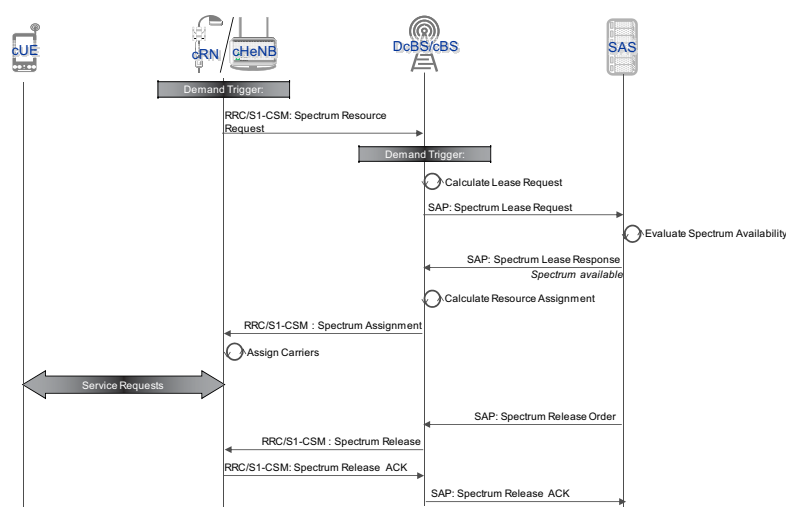

Fig. 8. Spectrum lease request procedure for both the $\mathrm{cRN}$ and the cHeNB in the Cell Spectrum Management (CSM) framework.

are collected from the $\mathrm{cRNs} / \mathrm{cHeNBs}$, the DcBS/cBS sends a message to the SAS indicating that the resources have been released and KPIs are reported for the spectrum usage of the cell.

c) CSM New Primary Alert Procedure: The purpose of this procedure is to notify the DcBSs/cBSs and associated cRNs/cHeNBs of a newly active primary operator so spectrum can be vacated. The procedure signaling diagram is shown in Figure 9. Using SAP, the primary operator issues a registration request to the SAS. This request contains information about the licensed spectrum, such as center frequency, bandwidth, and licensed geographic area. The SAS updates the geolocation database and returns a registration response. Using the geolocation database, the SAS then identifies and notifies the associated $\mathrm{DcBS} / \mathrm{cBS}$ that there is a new primary operator active on a specific channel. The cBSs then update their spectrum access rules, mark the channel as belonging to a primary operator, and determine new spectrum resource assignments. These new spectrum assignments are then sent to the cRNs/cHeNBs. Using these assignments, the cRNs/cHeNBs augment their respective carriers with the licensed spectrum to be used opportunistically. Each $\mathrm{cRN} / \mathrm{cHeNB}$ then sends an assignment $\mathrm{ACK}$ to the $\mathrm{DcBS} / \mathrm{cBS}$, to indicate the channels are vacated. After the channel is vacated, the cBS sends an ACK to the SAS. Once all of the cBSs have vacated the spectrum, the SAS notifies the primary operator.

\section{Domain Spectrum Management}

As with CSM, DSM provides local spectrum management of a geographic coverage area or domain. However, this domain could be a campus, stadium, or neighborhood in which a multitude of cHeNBs are deployed and managed by a cHeNB Gateway. Within this domain, the cHeNBs are assigned resources by the DSMgr, which resides on the cHeNB Gateway. Through a locally managed spectrum pool, the DSMgr assigns spectrum to $\mathrm{cHeNBs}$ within the domain. When the demand within the cell exceeds the available spectral capacity, the cHeNB Gateway, through its own SAC and the DSMgr, will send a lease request to obtain more spectrum for the spectrum pool. 


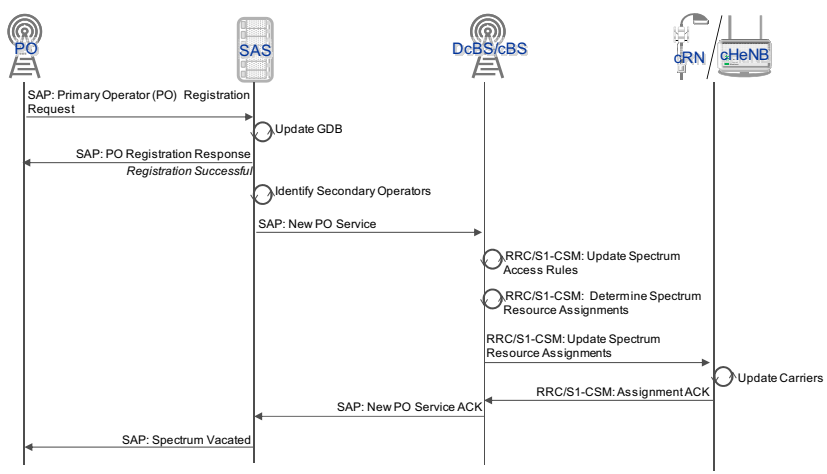

Fig. 9. New primary operators alerting procedure for the $\mathrm{cRN}$ and the $\mathrm{cHeNB}$ in the Cell Spectrum Management (CSM) framework.

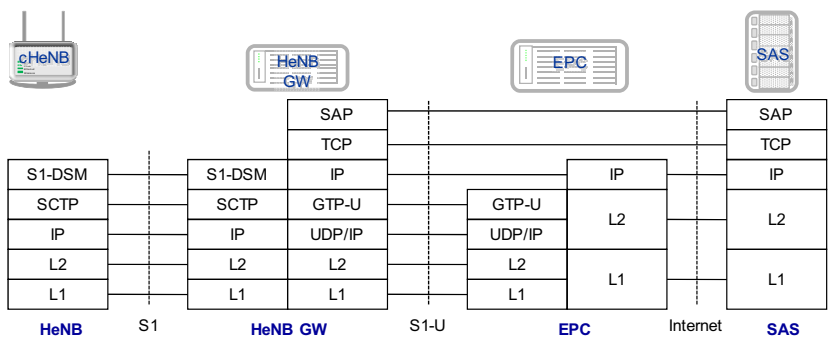

Fig. 10. S1-DSM signaling is used to locally manage the cHeNB within the domain of the cHeNB Gateway. Residing on the cHeNB Gateway, the Domain Spectrum Manager (DSMgr) translates orders and information to and from other $\mathrm{cBS}$ and the SAS.

1) DSM Interfaces: Similar to the CSMgr, the Domain Spectrum Manager (DSMgr) communicates directly with the cHeNB, using the S1-DSM protocol. The cHeNB Gateway uses its own SAC client, using the EPC as a relay and IP anchor to communicate with the SAS. Through the S1-DSM interface, the cHeNB sends resource requests and receives spectrum sensing orders to and from the cHeNB Gateway. These messages are translated into spectrum lease requests, using SAP, to the SAS, or cooperative sensing information to neighboring cBSs or cHeNB Gateway Domains. Spectrum sensing information is shared with other cHeNB Gateways or cBSs through the S1-CS link. These links are established using the neighbor discovery procedures based on [17]. The protocol stacks for each of these interfaces are shown in Figures 10 and 11 .

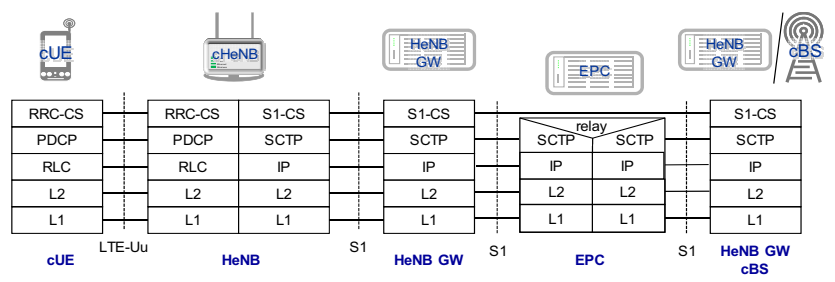

Fig. 11. S1-CS signaling is used to share spectrum sensing information among cHeNB Gateways and cBSs.

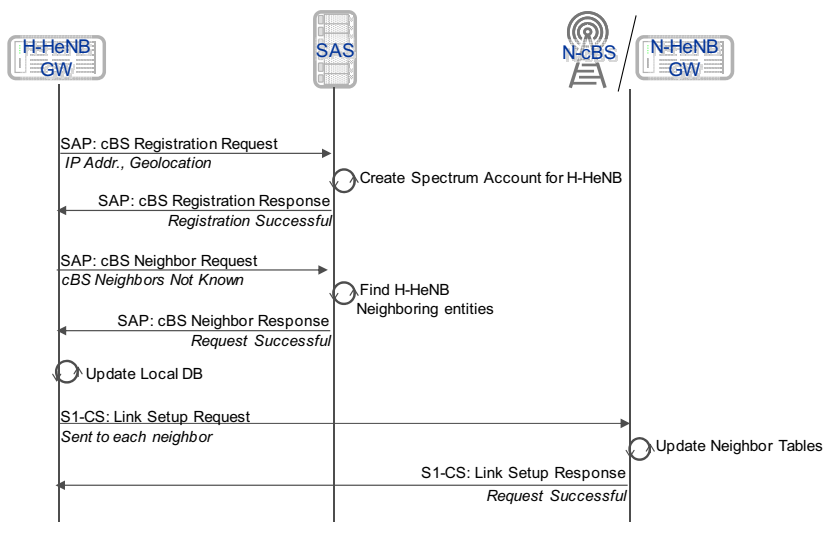

Fig. 12. cHeNB Gateway registers with the SAS, discovering the neighboring cHeNB Gateways and cBS

2) DSM Procedures: Like the CSMgr, the DSMgr translates orders and information from the cHeNB Gateway domain to other cBSs, cHeNB Gateways, and the SAS. Operationally, many of the procedures are similar to the ones adopted with CSM. The differences are primarily in the network elements exchanging messages and the S1-DSM and S1-CS interfaces. We illustrate these similarities and differences by introducing two modified procedures from [17]: registration/discover and cooperative sensing.

a) Registration/Discovery Procedure: The cBS Registration and Neighbor Discovery procedure is the genesis of all other procedures. When using SAP, all management entities must register with the SAS. Registration with the SAS is necessary for generating spectrum lease requests and discovering neighbors for the exchange of spectrum sensing information. Similarly, the cHeNB Gateway also registers with the SAS. This procedure is shown in Figure 12.

In the first SAP message, the DSM, on the Home-cHeNB Gateway (H-cHeNB Gateway), sends a registration request to the SAS. This registration request contains the IP address of the H-cHeNB Gateway, as well as geolocation information of the H-cHeNB Gateway domain. When this request is received at the SAS, the SAS creates a spectrum account for the $\mathrm{H}-\mathrm{cHeNB}$ Gateway and updates the geolocation database. After the SAS creates the H-cHeNB Gateway account, the SAS responds with a registration response, indicating that the registration was successful, and sends the spectrum access rule set based on the SAS policy. After registration is complete, the cHeNB Gateway then discovers the Neighboring-cBSs and also Neighboring-cHeNB Gateways through request/response signaling to the SAS. The SAS neighbor response contains the IP addresses of all the neighboring entities. Using the neighboring entities' IP addresses, the cHeNB Gateway then sends an S1-CS: Link setup request to support the exchange of sensing information.

b) Cooperative Sensing Procedure: In the DSM configuration, the cHeNB Gateways periodically collect spectrum sensing information from their own domain and share it with other cHeNB Gateways and cBSs. This procedure is illustrated 


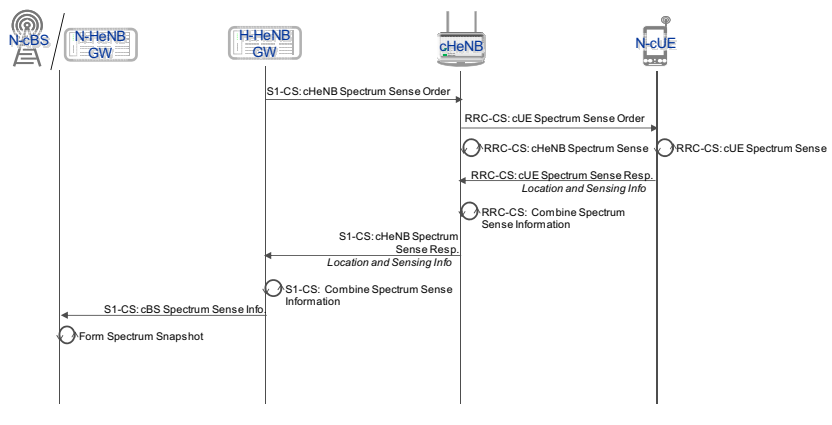

Fig. 13. cHeNB shares sensing information.

in Figure 13. The procedure begins with the H-cHeNB Gateway issuing the command to the cHeNB to collect sensing information. After receiving the spectrum sensing order, the cHeNB forwards this message to the cUEs and performs its own sensing. After receiving spectrum sensing information from all of the cUEs, the cHeNB then combines and reports this information to the $\mathrm{cHeNB}$ Gateway. After receiving all of the spectrum sensing information from all of the cHeNBs, the cHeNB Gateway then shares this information with the neighboring entities (i.e., N-cBSs and N-cHeNB Gateways) to form spectrum snapshots.

\section{Tradeoffs Between Architectures}

The choice of an appropriate management framework (SAC, CSM, or DSM) for a DSA HetNet depends on the usage scenario. If a substantial number of $\mathrm{cHeNBs}$ and $\mathrm{RNs}$ are deployed in a SAC configuration, the number of spectrum lease requests could potentially overload the EPC and the SAS. Considering this disadvantage, SAC makes more sense when the cHeNBs or RNs are deployed sparsely. In the case of dense deployments or a substantial number of $\mathrm{cHeNBs}$ and RNs, the CSM or DSM configurations clearly impose the least overhead. In a suburban setting, cHeNBs may be deployed within households and perhaps have some macrocell coverage. In this case, cHeNB deployments would be less dense than in an institutional deployment, such as what would be found at a campus or a stadium. Thus, CSM may make more sense in a suburban setting, because spectrum management of a macro-cell coverage area is already being performed. In the case of densely deployed cHeNBs, DSM may be preferable to CSM, since signaling from densely deployed cHeNBs may overload the local CSMgr. Signaling loads could be balanced through the use of cHeNB Gateways and DSMgrs. We envision that future HetNet deployments which use DSA will likely adopt a combination of these different frameworks to meet the needs of their local markets.

\section{Performance Evaluation}

In the previous section, we presented the affected control planes, new network elements, operational procedures, and our three proposed DSA management frameworks to support a DSA overlay in an LTE+ HetNet network. In this section, we formulate integer programs to assess the benefits of using
DSA in an LTE+ HetNet. We compare an LTE+ network with the SA framework and a DSA HetNet management framework (i.e., SAC, CSM, or DSM) to one without. In considering this network, licensed and opportunistic spectrum is aggregated to form carriers to meet the traffic demand.

In our first formulation, we seek to establish a baseline. To this end, we first formulate an integer program such that only licensed spectrum is available to an LTE+ HetNet, which includes cHeNBs and cBSs. In our second formulation, we allow the cBSs to use only licensed spectrum and allow the cHeNBs to use DSA and licensed spectrum. We compare the optimal solutions of each of these integer programs to understand the benefits of using DSA spectrum in a LTE+ HetNet. The objective in every case is to maximize the assignment of the channels onto the carriers of the cBSs and cHeNBs such that the demand is met.

\section{A. Problem Formulations}

In our first formulation, we consider an LTE+ HetNet with $W$ licensed channels ${ }^{1}$, which can be deployed throughout a network with $B$ cBSs and $H$ cHeNBs. To manage intercell interference between $\mathrm{cBS}$, channels are aggregated into cell-edge or cell-center carriers [20], where channels in the cell-edge carriers must be different among neighboring base stations. This frequency/channel assignment forms the first of our assignment constraints. Additionally, we prevent inter-tier interference by assuming orthogonal channel assignment of LTE+ channels between cHeNB and cBS. cHeNB deployments should also avoid inter-cell interference by assigning orthogonal frequencies between neighbors.

Let $x_{i, c, k}=1$ when $\mathrm{cBS} i$ assigns channel $c$ to carrier $k$, where $k=1$ and $k=2$ indicate a cell-center and a celledge carrier, respectively. Denote $d_{i, k}$ as the demand in number of channels at cBS $i$ on carrier $k$ and $d_{h}$ as the demand, in number of channels, at cHeNB $h$. Through an adjacency matrix $\mathbf{N}$, we define neighbor relationships between cBSs, where $n_{i, j}=1$ indicates cBS $i$ is a neighbor to $\mathrm{cBS} j$. cHeNBs follow similar assignment rules. Through an adjacency matrix M, we define neighbor relationships between cHeNBs, where $m_{h, g}=1$ indicates cHeNB $h$ is a neighbor to cHeNB $g$. To manage inter-tier interference (i.e., interference between a cHeNB and a cBS) cHeNBs within a cell-center or cell-edge cannot use channels contained in those respective carriers. Let $u_{h, c}=1$ to indicate when cHeNB $h$ uses channel $c$. Through an interference matrix $\mathbf{Q}$, we define inter-tier relationships, where $q_{i, h, k}=1$ indicates $\mathrm{cBS} i$, carrier $k$ overlaps with the coverage of cHeNB $h$. Here we introduce our first formulation, $\mathcal{F}_{1}$, which uses only licensed channels to satisfy the demands of the network given the constraints described above.

\footnotetext{
${ }^{1}$ In LTE+, a resource block is based on $180 \mathrm{kHz}$.
} 
Maximize:

$$
\sum_{i=1}^{B} \sum_{c=1}^{W} \sum_{k=1}^{2} x_{i, c, k}+\sum_{h=1}^{H} \sum_{c=1}^{W} u_{h, c}
$$

Subject to:

$$
\begin{array}{ll}
\sum_{c=1}^{W} x_{i, c, k} \leq d_{i, k} & \forall i, k \\
\sum_{c=1}^{W} u_{h, c} \leq d_{h} & \forall h \\
\sum_{k=1}^{2} x_{i, c, k} \leq 1 & \forall i, c \\
n_{i, j}\left(x_{i, c, 2}+x_{j, c, 2}\right) \leq 1 & \forall i, j, c \\
m_{g, h}\left(u_{h, c}+u_{g, c}\right) \leq 1 & \forall g, h, c \\
q_{i, h, k}\left(x_{i, c, k}+u_{h, c}\right) \leq 1 & \forall i, h, k, c \\
x_{i, c, k}, u_{h, c} \in\{0,1\} & \forall i, c, k
\end{array}
$$

Our objective function is to maximize the number of channels assigned to each cBS and $\mathrm{cHeNB}$ in Function (1) using only licensed channels. In Constraints (2) and (3), channels are only assigned as required to meet demand. Constraint (4) enforces that channels should only be used once within the cBS cell (i.e., cell-edge and cell center carriers cannot have the same channel). To prevent inter-cell interference, Constraints (5) and (6) prevent neighboring cBS cell-edges and cHeNB cells from using the same channels. Constraint 7 is used to prevent intertier interference.

To develop our second formulation, we extend formulation $\mathcal{F}_{1}$ by considering a HetNet that can use licensed and opportunistic spectrum. Specifically, we consider opportunistic use of TV white spaces and unused GSM channels ${ }^{2}$. For TV white spaces we consider network elements that consult and report to the SAS for determining availability. To integrate this into our model, we define an interference matrix $\mathbf{P}$, where $p_{i, l}=1$ if cBS cell $i$ is not within interfering distance of TV station $l$. We further define $y_{i, l, c, k}=1$ to indicate when cBS $i$ uses TV station $l$ 's channel $c$ on carrier $k$, where there are $P$ TV stations, each with $V$ channels available.

Furthermore, we consider the case where GSM channels available at the local cell can be opportunistically reused by the cell for LTE+. In this case, we consider sensing at the cell to determine GSM channel availability. We also consider the case where GSM spectrum is already deployed such that interference between cells has been mitigated (i.e., no adjacent cell interference). Thus, we denote matrix $\mathbf{A}$, where $a_{i, c}=1$ if the base station $i$ 's channel $c$, from the GSM spectrum, is available for secondary use. We further denote $z_{i, c, k}=1$ to indicate when cBS $i$ uses channel $c$, from the GSM spectrum, on carrier $k$, where each base station has $U$ potential channels available.

\footnotetext{
${ }^{2}$ We consider a $200 \mathrm{kHz}$ spectrum block as an opportunistic LTE+ channel.
}

Similarly, for the cHeNB, we define an interference matrix $\mathbf{S}$, where $s_{h, l}=1$ if $\mathrm{cHeNB} h$ is not within interfering range of TV station $l$. We further denote $v_{i, l, c}=1$ to indicate when cHeNB $i$ uses TV station $l$ 's channel $c$. GSM channels available at the local cell can also be used on an opportunistic basis when unoccupied. Further denote $w_{h, i, c}=1$ to indicate when cHeNB $h$ uses channel $c$, from the GSM cell $i$, where each base station has $U$ potential channels available. We assume that GSM frequencies are previously deployed to minimize inter-cell interference, and therefore, GSM channels can only be opportunistically used within the same cell. We also assume that the GSM and LTE+ base stations are collocated within the same cell and share the same coverage area. Given our described constraints we present our second formulation, $\mathcal{F}_{2}$.

Maximize:

$$
\begin{aligned}
& \text { MaxChans }=\sum_{i=1}^{B} \sum_{c=1}^{W} \sum_{k=1}^{2} x_{i, c, k}+\sum_{i=1}^{B} \sum_{l=1}^{P} \sum_{c=1}^{V} \sum_{k=1}^{2} y_{i, l, c, k}+ \\
& \sum_{i=1}^{B} \sum_{c=1}^{U} \sum_{k=1}^{2} z_{i, c, k}+\sum_{h=1}^{H} \sum_{c=1}^{W} u_{h, c}+\sum_{h=1}^{H} \sum_{l=1}^{P} \sum_{c=1}^{V} v_{h, l, c}+ \\
& \sum_{h=1}^{H} \sum_{c=1}^{U} w_{h, i, c}
\end{aligned}
$$

Subject to:

$$
\begin{aligned}
& \sum_{c=1}^{W} x_{i, c, k}+\sum_{c=1}^{V} \sum_{l=1}^{P} y_{i, l, c, k}+\sum_{c=1}^{U} z_{i, c, k} \leq d_{i, k} \quad \forall i, k \\
& \sum_{c=1}^{W} u_{h, c}+\sum_{l=1}^{P} \sum_{c=1}^{V} v_{h, l, c}+\sum_{c=1}^{U} w_{h, i, c} \leq d_{h} \quad \forall h \\
& \sum_{k=1}^{2} x_{i, c, k} \leq 1 \\
& \forall i, c \\
& \sum_{k=1}^{2} y_{i, l, c, k} \leq p_{i, l} \\
& \forall i, l, c \\
& \sum_{k=1}^{2} z_{i, c, k} \leq a_{i, c} \\
& \forall i, c \\
& v_{h, l, c} \leq s_{h, l} \\
& \forall h, l, c \\
& w_{h, i, c} \leq a_{i, c} \sum_{k=1}^{2} q_{i, h, k} \\
& \forall h, c, i \\
& n_{i, j}\left(x_{i, c, 2}+x_{j, c, 2}\right) \leq 1 \\
& \forall i, j, c \\
& n_{i, j}\left(y_{i, l, c, 2}+y_{j, l, c, 2}\right) \leq 1 \\
& \forall i, j, l, c \\
& m_{g, h}\left(u_{h, c}+u_{g, c}\right) \leq 1 \\
& \forall \mathrm{g}, h, c \\
& m_{g, h}\left(v_{h, l, c}+v_{g, l, c}\right) \leq 1 \\
& \forall g, h, l, c \\
& m_{g, h}\left(w_{h, i, c}+w_{g, i, c}\right) \leq 1 \\
& \forall g, h, i, c \\
& q_{i, h, k}\left(x_{i, c, k}+w_{h, i, c}\right) \leq 1 \\
& \forall i, h, k, c \\
& q_{i, h, k}\left(y_{i, l, c, 2}+v_{h, l, c}\right) \leq 1 \\
& \forall i, h, k, c \\
& q_{i, h, k}\left(z_{i, c, k}+v_{h, l, c}\right) \leq 1 \\
& \forall i, h, k, c
\end{aligned}
$$




$$
\begin{array}{ll}
\sum_{l=1}^{P} \sum_{c=1}^{V} \sum_{k=1}^{2} y_{i, l, c, k} \leq \sum_{l=1}^{P} p_{i, l} V & \forall i \\
\sum_{l=1}^{P} \sum_{c=1}^{V} v_{h, l, c} \leq \sum_{l=1}^{P} s_{h, l} V & \forall h \\
\sum_{c=1}^{U} \sum_{k=1}^{2} z_{i, c, k} \leq U & \forall i \\
x_{i, c, k}, y_{i, l, c, k}, z_{i, c, k}, u_{h, c}, v_{h, l, c}, w_{h, i, c} \in\{0,1\} & \forall i, l, h, c, k
\end{array}
$$

In $\mathcal{F}_{2}$, we carry the constraints introduced in $\mathcal{F}_{1}$ and introduce the new equivalent constraints for inter-cell interference, which consider DSA. The objective function in Equation (9) maximizes the assignment of licensed and DSA channels for the entire HetNet. Constraints (10) and (11) prevent the assignments of spectrum channels beyond the associated demand for the cBS and cHeNB, respectively. Similar to Constraint (4), the assignment of the same TV white space and GSM channels to different cBS carriers within the same cell is prevented through Constraints (12) and (13), respectively. For defining permitted assignments of TV white space and GSM spectrum for cHeNB, we use Constraints (14) and (15), respectively. To prevent the inter-cell and inter-femto-cell interference of DSA channels, we use Constraints (16) - (18) to define additional neighbor relationships. Inter-tier interference of DSA channels is similarly addressed with Constraints (19) and (20). Finally, Constraints (21)-(23) define the limits of available DSA channels for the cBS and cHeNB. Next, we discuss our input scenarios in which we can compare optimal solutions to understand the quantitative benefits of using DSA in LTE+ HetNets.

\section{B. Simulated Scenarios and Results}

Our primary goal in creating our scenarios was to create the most realistic input possible for our performance analysis. We selected the Blacksburg-Christiansburg, VA metro-area as the basis for our study and began the arduous task of gathering and processing the necessary GIS data from many different sources. We gathered data from the FCC transmitter databases, building footprints from the city of Blacksburg and Christiansburg, and population data from Oak Ridge National Lab's LandScan [21]. Using this GIS data, we create a HetNet topology and an associated representative user population for inputs to our mathematical formulations. To create hybrid carriers, we consider dynamic 200kHz LTE+ channels from licensed and opportunistic spectrum (i.e.,GSM and TV white spaces) and use FCC allocated bandwidths for determining total available spectrum.

1) Topology: Our HetNet comprises two tiers, the macrocell layer, supported by the cBS, and the femto-cell layer supported by the cHeNB. We model the macro-cell layer of our HetNet using real locations of cellular base stations within our study area. We retrieved a list from the FCC's Antenna Structure Registration (ASR) database [22] and identified registrations to known cellular network providers and tower management companies. Additionally, we checked our list against

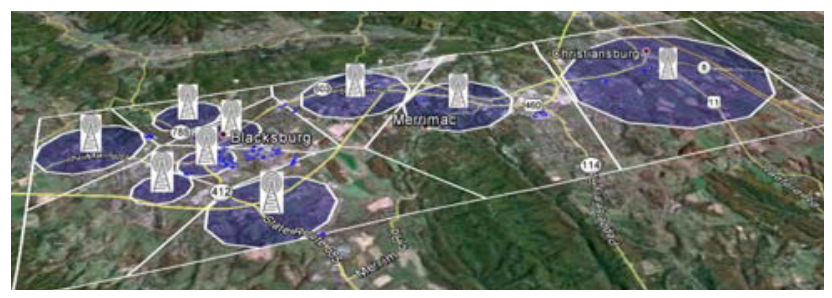

Fig. 14. Rendered view of our study area using real locations of base stations. Edge coverage areas are approximated through use of Voronoi cells and Cellcenter coverage is determined by a maximal circumference within the Voronoi macro-cell.

satellite imagery to determine whether the site contained cellular equipment. We also visually identified additional sites, not registered in the ASR, and added them to our list. Determining coverage areas of these sites is problematic, because this information is proprietary. Therefore, we use Voronoi cells ${ }^{3}$ to approximate macro-cell coverage areas [23]. Using Voronoi cells also allows us to determine adjacency information for our constraints in the mixed integer linear programs discussed in the previous section. For cell-center coverage areas, we consider a maximal circular coverage area within each Voronoi macro-cell. A rendered view of our macro-cell layer and study area is shown in Figure 14. To create a realistic femtocell layer for our HetNet, we overlay population information, from LandScan, with building footprints. LandScan provides the finest resolution of population distribution available by providing an ambient population count ${ }^{4}$ in square kilometer pixels. Through our overlay, we identify buildings which lie in areas where the ambient population count exceeds 300. For each of these buildings, we estimate the number of femtocells required for each building by dividing the building area the approximate coverage area of a femto-cell $\left(\pi(100 \mathrm{ft})^{2}=\right.$ $31 k f t^{2}$ ), then place femto-cells in these buildings according to a random distribution. As with the macro-cells, femto-cell coverage areas and adjacency relationships are determined through Voronoi cells. The entire femto-cell layer is twodimensional, i.e., at the moment we do not consider the effects of femto-cell deployments in buildings with multiple floors. An example of an in-building deployment of our femto-cell layer in shown in Figure 15.

2) Channel Demand: Channel demand for the $\mathrm{cBS} d_{i, k}$ and the cHeNB $d_{h}$ is calculated by:

$$
d=\left\lceil\frac{1}{1000 \tau} \sum_{i}^{P}\left\lceil 1000 \frac{\Delta_{i}}{\theta\left(\gamma_{i}\right)}\right\rceil .\right.
$$

By Equation (24), each individual user $i$ of cell population $P$, demands $\Delta_{i}$ bits. The LTE + channel throughput mapping function $\theta$ translates user $i$ 's Signal to Interference and Noise Ratio (SINR) $\gamma_{i}$ into an available data rate for a single LTE+ channel. The quotient of $\Delta_{i}$ and $\theta$ yields the number

\footnotetext{
${ }^{3}$ Each facet of the Voronoi cells represents a set of equidistant points between base station sites.

${ }^{4}$ The ambient population count is a metric that incorporates both diurnal movements and collective travel habits.
} 


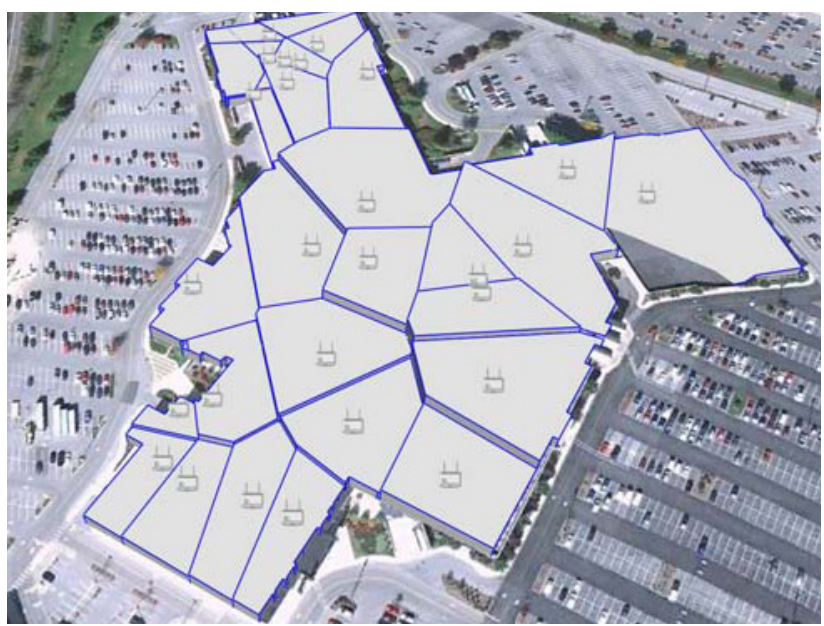

Fig. 15. An example of an in-building deployment of our femto-cell layer. Polygons represent femto-cell coverage within a building. Locations of cHeNBs placement are indicated by the icons.

of seconds for which a single channel will be required by user $i$. Furthermore, in LTE+ a single channel is broken into $1 \mathrm{~ms}$ scheduling blocks [20]. Thus, we convert the argument within the summation from seconds into scheduling blocks by multiplying by 1000 . To convert the sum into the total number of channels, we bound the amount of time a channel can provide resources by a time period $\tau$, e.g., if the channel is bounded by 2 seconds it can provide 2000 resource blocks.

$P$ for the cell is determined through a count of users placed within the service area of the respective polygon. We place $90 \%$ of the pixel population in buildings and randomly place the remaining population within the pixel. Cell populations are determined by counting the number of placements within the respective polygons. To model $\Delta_{i}$, we consider HyperText Transfer Protocol (HTTP) responses, i.e., a user downloading a web page. Following [24], we model the length of HTTP responses using the Pareto distribution. The Pareto distribution probability density function is given by:

$$
p(x)=\sigma k^{\sigma} x^{-\sigma-1}, \quad \sigma, k>0, \quad x \geq k,
$$

with parameters from [24], $\sigma=1.06$ and $k=8000$ bits.

The channel throughput mapping function $\theta$ is given in Table I. We derived $\theta$ using the SINR to Channel Quality Indicator (CQI) mapping function from [25], and use the CQI to determine and modulation and coding rates using [26]. Using the modulation and coding rates, we calculate $\theta$ to represent the throughput for a single LTE+ channel. The SINR user $i \gamma_{i}$ is modeled using a lognormal random variable.

3) Spectrum and DSA: In our scenario, cBSs and cHeNBs are allowed to aggregate spectrum channels to form carriers in the network. LTE+ channels can be dynamically assigned to any cBS or cHeNB using licensed, GSM, or TV white space spectrum. The FCC bands outlined in Table II are considered for use in the network. The GSM spectrum availability for LTE+ channels is modeled by the modified beta distribution, as used in [17] and we consider a frequency reuse factor of

\begin{tabular}{|c|c|c|c|}
\hline SINRmin & Modulation & ECR & $\theta$ \\
\hline-8.93 & 4QAM & 0.08 & 27432 \\
\hline-6.87 & 4QAM & 0.12 & 42192 \\
\hline-4.80 & 4QAM & 0.19 & 67860 \\
\hline-2.73 & 4QAM & 0.30 & 108288 \\
\hline-0.67 & 4QAM & 0.44 & 157860 \\
\hline 1.40 & 4QAM & 0.59 & 211644 \\
\hline 3.47 & 16QAM & 0.37 & 265752 \\
\hline 5.53 & 16QAM & 0.48 & 344520 \\
\hline 7.60 & 16QAM & 0.60 & 433152 \\
\hline 9.67 & 64QAM & 0.46 & 491508 \\
\hline 11.73 & 64QAM & 0.55 & 597996 \\
\hline 13.80 & 64QAM & 0.65 & 702432 \\
\hline 15.87 & 64QAM & 0.75 & 814212 \\
\hline 17.93 & 64QAM & 0.85 & 920700 \\
\hline 20.00 & 64QAM & 0.93 & 999864 \\
\hline
\end{tabular}

TABLE I

SINR TO THROUGHPUT MAPPING FUNCTION $\theta$

\begin{tabular}{|c|c|c|c|}
\hline Band & Net & Freq Bands(MHz) & BW(MHz) \\
\hline Cellular & GSM & $824-849,869-894$ & 25 \\
\hline AWS & GSM & $1710-1755,2110-2155$ & 45 \\
\hline PCS & GSM & $1850-1910,1930-1990$ & 60 \\
\hline $700 \mathrm{MHz}$ & LTE & $698-716,775-788,805-806$ & 32 \\
\hline TV & TV & $512-608,614-698$ & 84 \\
\hline
\end{tabular}

TABLE II

AsSUMED LICENSED SPECTRUM ALLOCATION FOR GSM AND LTE+ NETWORKS.

4. For TV white spaces, we retrieved TV contour areas from the FCC's Consolidated Database System (CDBS) [27]. From the CDBS, we identified 12 stations which overlapped our area. In our scenario, any cell which overlaps a TV contour is not permitted to operate in the associated TV spectrum. Additionally, we also consider frequency division duplexing, i.e. paired frequencies in the up-link and down-link.

\section{Results}

Using our scenarios as input to our formulations, we assume $\tau=1 s$, vary the probability of active users and allow the LTE+ network to experience increasing demand. To solve our maximization problems we used the Matlab Parallel Computing Toobox [28], CPLEX [29], and used the multi-core (832) Advanced Research Computing servers at Virginia Tech [30] (Ithaca) to run 1000 simulations per data point. We used both MacroNets and HetNet topologies for our simulations and adjust our optimization model accordingly. Additionally, we examine how the use of different spectrum bands increase the proportion of traffic served, where the proportion of traffic served is determined by the following equation:

$$
\text { ProportionalTraffic }=\frac{\text { MaxChans }}{\sum_{i=1}^{B} \sum_{k=1}^{2} d_{i, k}+\sum_{h=1}^{H} d_{h}} .
$$




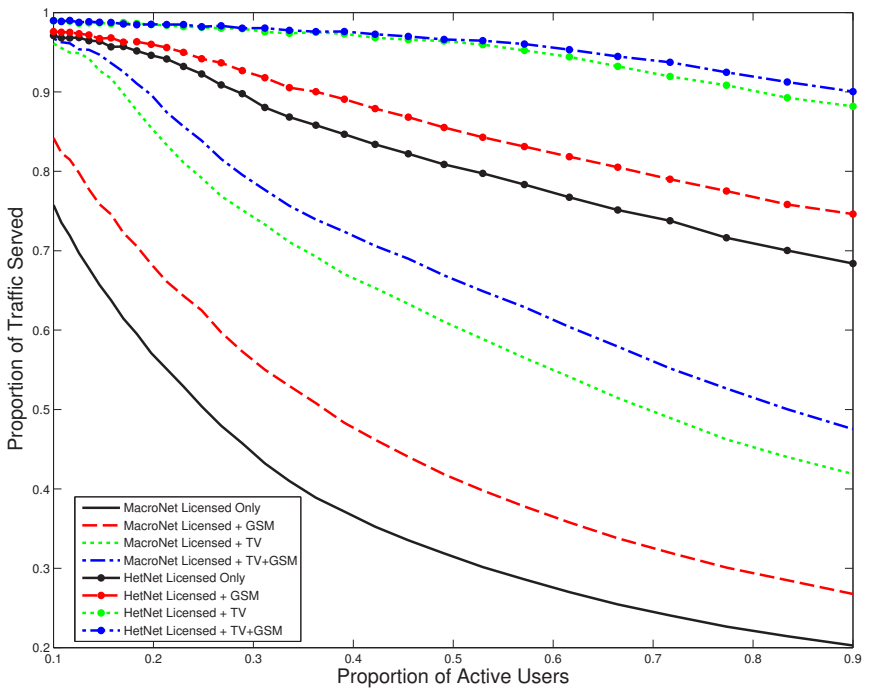

Fig. 16. The use of HetNets and additional spectrum are both required to accommodate the highest grade of service.

Our results are shown in Figure 16. The most salient feature of this figure is the difference between traffic served for the MacroNet and the HetNet. When comparing the Licensed Only curves of the MacroNet and HetNet there is an average increase of $41 \%$ over the performance of the MacroNet. Additionally, we observe TV white spectrum provides the largest average gain in performance for both Nets: $27 \%$ for MacroNet and $10 \%$ increase for the HetNet. Although the gains in using opportunistic use of GSM is modest, $10 \%$ and $2 \%$ for the Macro and HetNet, respectively, we believe that these gains will significantly increase as operators continue to migrate users to LTE+. While we see increase in performance with additional spectrum and use of HetNets, we note that network operators usually desire a high grade of service for their networks. Typically, network operators desire a blocking percentage less than $5 \%$. If we were to apply this performance criterion, we note that to cope with additional demand networks will require both additional spectrum and smaller cells. We conclude by emphasizing that although spectrum availability varies by geography, opportunistic spectrum may provide substantial performance gains in cellular markets.

\section{Conclusion and Future Work}

Our work has examined and proposed future methods by which an LTE+ HetNet can deploy a DSA overlay. In the first part of our paper, we proposed three new management frameworks for DSA in HetNets: Spectrum Accountability Client, Cell Spectrum Management, and Domain Spectrum Management. For these spectrum management frameworks, we defined protocol interfaces and operational signaling scenarios to support cooperative sensing, spectrum lease management, and alarm scenarios for rule adjustment. In the final section of the paper, we formulated integer programs to illustrate the quantitative benefits of deploying DSA spectrum in an LTE+ HetNet. Using GIS data, we modeled a realistic HetNet as an input scenario for our integer programs. In summary, this paper proposed architectural frameworks for the deployment of DSA in HetNets and also quantified those benefits through an optimization model using integer programs. Through this work, future LTE+ standards, infrastructure vendors, and network operators can gain a better understanding of the effects and benefits of using DSA in HetNets.

We have identified two branches for the extension of the work presented here. First, although our formulations were useful in illustrating the quantitative benefits of using DSA, our formulations did not consider the performance tradeoffs between each of the management frameworks that we proposed. We are in the process of extending this work by performing this comparison through extensions of our mathematical formulations. In these extensions, we envision a method that uses sets of cascading mathematical programs to represent each of the management frameworks that we proposed. Second, the use of GIS population data and known transmitter locations from the FCC provides many opportunities for critical research needed by policy makers. Specifically, we are interested in studies which examine TV band interference from opportunistic use. Additionally, we see a need for a nationwide spectrum demand and inventory study, which includes TV white spaces and National Telecommunications and Information Administration (NTIA) spectrum proposed for opportunistic use. In this second branch of research, we believe results can bring great benefits in advancing DSA policy to aid the forthcoming spectrum crisis.

\section{REFERENCES}

[1] Global Mobile Suppliers Association, "Evolution to LTE report," GSM/3G Market/Technology Update, May 2011.

[2] Federal Communications Commission, "The benefits of additional spectrum," U OBI Technical Paper, no. 6, Oct 2010.

[3] R. Engelman, K. Abrokwah, and G. Dillon, "Report of the spectrum efficiency working group," Federal Communications Commission Spectrum Policy Task Force, Nov 2002.

[4] Federal Communications Commission, " In the Matter of: Unlicensed Operation in the TV Broadcast Bands (ET Docket No. 04-186) and Additional Spectrum for Unlicensed Devices Below $900 \mathrm{MHz}$ and in the $3 \mathrm{GHz}$ Band (ET Docket No. 02-380)," FCC 10-174:Second Memorandum Opinion and Order, September 2010.

[5] Ofcom, "Implementing geolocation," Consultation, November 2010.

[6] S. Parkvall, E. Dahlman, A. Furuskar, Y. Jading, M. Olsson, S. Wanstedt, and K. Zangi, "LTE-advanced-evolving LTE towards IMT-advanced," IEEE 68th Vehicular Technology Conference (VTC), 2008.

[7] W. Lehr and J. Chapin, "Hybrid Wireless Broadband," 37th Research Conference on Communication, Information and Internet Policy (TPRC), 2009.

[8] Informa Telecoms and Media, "Global market shares," 4G Americas Statistics, March 2011, http://www.4gamericas.org/. 
[9] V. Blaschke, C. Kloeck, J. Weiss, T. Renk, and F. Jondral, "Opportunistic WiMAX-GSM coexistence," Communications, IET, July 2008.

[10] V. Chandrasekhar and J. Andrews, "Spectrum allocation in tiered cellular networks," Communications, IEEE Transactions on, vol. 57, no. 10, pp. 3059-3068, 2009.

[11] D. López-Pérez, A. Ladányi, A. Juttner, and J. Zhang, "OFDMA femtocells: A self-organizing approach for frequency assignment," Personal, Indoor and Mobile Radio Communications, 2009 IEEE 20th International Symposium on, pp. 2202-2207, 2009.

[12] D. López-Pérez, A. Valcarce, G. De La Roche, and J. Zhang, "OFDMA femtocells: a roadmap on interference avoidance," Communications Magazine, IEEE, vol. 47, no. 9, pp. 41-48, 2009.

[13] L. Garcia, K. Pedersen, and P. Mogensen, "Autonomous component carrier selection: interference management in local area environments for lte-advanced," IEEE Communications Magazine, vol. 47, no. 9, 2009.

[14] D. López-Pérez, I. Guvenc, G. de la Roche, M. Kountouris, T. Quek, and J. Zhang, "Enhanced intercell interference coordination challenges in heterogeneous networks," Wireless Communications, IEEE, vol. 18, no. 3, pp. 22-30, 2011.

[15] G. Gur, S. Bayhan, and F. Alagoz, "Cognitive femtocell networks: an overlay architecture for localized dynamic spectrum access [dynamic spectrum management]," Wireless Communications, IEEE, vol. 17, no. 4, pp. 62-70, 2010.

[16] S. Al-Rubaye, A. Al-Dulaimi, and J. Cosmas, "Cognitive femtocell," IEEE Vehicular Technology Magazine, vol. 6, no. 1, pp. 44-51, 2011.

[17] J. Deaton, R. Irwin, and L. DaSilva, "The Effects of a Dynamic Spectrum Access Overlay in LTE-Advanced Networks," IEEE 6th International Symposium on New Frontiers in Dynamic Spectrum Access Networks (DySPAN), May 2011.

[18] 3rd Generation Partnership Project, "TS 36.300 V10.3.0," Evolved Universal Terrestrial Radio Access (E-UTRA) and Evolved Universal Terrestrial Radio Access Network (E-UTRAN) Overall description Release 10, March 2011.

[19] —_, "TS 23401 V10.3.0," General Packet Radio Service (GPRS) enhancements for Evolved Universal Terrestrial Radio Access Network (E-UTRAN) Release 10, June 2011.

[20] R. Kwan and C. Leung, "A survey of scheduling and interference mitigation in LTE," Hindawi Journal of Electrical and Computer Engineering, 2010.

[21] Oak Ridge National Lab, 2011, http://www.ornl.gov/sci/landscan.

[22] Federal Communications Commission, Antenna Structure Registration Database, 2011, http://wireless2.fcc.gov/UlsApp.

[23] A.-E. Baert and D. Seme, "Voronoi mobile cellular networks: topological properties," July 2004.

[24] B. Mah, "An empirical model of HTTP network traffic,"
Sixteenth Annual Joint Conference of the IEEE Computer and Communications Societies (INFOCOM'97), April 1997.

[25] J. Ikuno, M. Wrulich, and M. Rupp, "System level simulation of LTE networks," 2010 IEEE 71st Vehicular Technology Conference (VTC), 2010.

[26] 3rd Generation Partnership Project, "TS 36.213 V10.4.0," Evolved Universal Terrestrial Radio Access (E-UTRA);Physical layer procedures(Release 10), December 2011.

[27] Federal Communications Commission, TVQ TV Database Query, 2011, http://transition.fcc.gov/mb/video/tvq.html.

[28] Matlab, "Parallel Computing Toolbox," 2011, http://www.mathworks.com/products/parallelcomputing/.

[29] IBM, "ILOG CPLEX Optimizer," 2011, http://www01.ibm.com/software/integration/optimization/cplexoptimizer/.

[30] Advanced Research Computing, 2011, http://www.arc.vt.edu. 